Supporting Information

\title{
Mechanical Adaptability of Patterns in Constrained Hydrogel Membranes
}

\author{
Yao Xiong and Olga Kuksenok ${ }^{1, *}$ \\ ${ }^{1}$ Department of Materials Science and Engineering, Clemson University, Clemson, South Carolina 29634, \\ United States \\ ${ }^{*, 1}$ : okuksen@clemson.edu
}

Section S1: Additional simulations details and analysis

p. S2
A. Applying random perturbations
B. Calculating Poisson's ratio
C. Linear stability analysis.

Section S2: Supplementary figures

p. S4

Figure S1. Equilibrium volume fraction of polymer, $\phi$, as a function of temperature, $T$

Figure S2. Time evolution of patterns upon the temperature quench

Figure S3. Effects of random perturbations and sample length

Figure S4. Characteristic ratio $\alpha$ as a function of the depth of the temperature quench

Figure S5. Effects of the sample width and crosslink density on the wavelength and the amplitude Figure S6. Poisson's ratio of PNIPAAm gels

Figure S7. Linear stability analysis of the flat confined elastic plate

Figure S8. Effects of the sample thickness

Figure S9. The average sample thickness prior to the onset of pattern formation

Figure S10. Patterns characteristics under stretching and compression cycles

Figure S11. Dynamics of patterns during an equilibrated cycle

Figure S12. Effects of the sample length

Figure S13. Dynamics during slow stretching and compression $\left(\dot{\gamma}=5 \times 10^{-5}\right)$

\section{Section S3: Supplementary Tables}

Table S1. Values of $\alpha_{c}, p_{1}$, and $p_{2}$ for dashed fitting curves in Figure S5b.

Section S4: References

p. S13 


\section{Section S1: Additional simulations details and analysis}

A. Applying random perturbations. In all independent simulations involving the temperature quench, we applied small random perturbations during the fixed time interval $(\delta t=100)$. We first probed four different scenarios of perturbing an initially equilibrated system (Figures S3a-b). In Cases 1-3, we locally varied the temperature within the ten percent of elements, which were randomly selected for each independent run. The simplest means to randomly perturb the size of the element mimicking local perturbations in the system $^{1}$ is to vary external conditions for the short period of time; to do so, we modified the temperature of the elements locally for a fixed period of time. We probed three different amplitudes of the decrease in temperature in the independent simulation runs: $\delta T=-0.05^{\circ} \mathrm{C}(\mathrm{Case} 1),-0.1^{\circ} \mathrm{C}(\mathrm{Case} 2)$, and $-0.5^{\circ} \mathrm{C}$ (Case3). The decrease in temperature was chosen since it results in the local swelling of the selected elements. In Case 4, we applied random perturbations to each element within the sample during the same time interval $(\delta t=100)$ with the amplitude of the temperature change of $\delta T=0.1^{\circ} \mathrm{C}$. In this case, the local perturbation in $T$ was chosen as a randomly fluctuating variable with Gaussian statistics and zero mean. A maximum local change in the degree of swelling of the elements due to these perturbations ranged between $0.12 \%$ (Case 1) and $1.22 \%$ (Case 3 ) with respect to the samples with no perturbations applied. Figures S3a$\mathrm{b}$ show that applied random perturbations for all the cases considered do not affect the equilibrium patterns characteristics, however, as anticipated, without the applied noise buckling was not observed at the most shallow depths of the temperature quench (black lines in Figures 3a-b). Herein, for all the simulations under the temperature quench, we set the random perturbations as defined in Case2.

B. Calculating Poisson's ratio. To calculate the Poisson's ratio of the sample as a function of temperature, we run a series of simulations for the samples undergoing uniaxial compression at a range of temperatures. In this series of simulations, a compressive strain with the magnitude $\varepsilon_{\|}=0.1$ was applied to the sample of a size of $51 \times 11 \times 11$ nodes along its length (in the $x$-direction, as shown by the arrow in Figure S6) at various temperatures between $16^{\circ} \mathrm{C}$ and $40^{\circ} \mathrm{C}$ (herein, we set $\phi_{0}=0.114$ and $c_{0}=4 \times 10^{-3}$, which corresponds to the reference parameters set). Specifically, we assigned the boundary nodes at $x=l_{0}$ a low velocity $\left(4.9 \times 10^{-3}\right)$ along the "- $x$ "-direction while the longitudinal positions of nodes at $x=0$ were held constant. Free transverse motion of all the nodes was permitted. When the strain in the direction of the uniaxially applied deformation (as indicated by the arrow in the snapshot in Figure S6) reached $\varepsilon_{\|}=0.1$, the motion was halted. The Poisson's ratio was calculated as $v=-\varepsilon_{\perp} / \varepsilon_{\|}$, where $\varepsilon_{\perp}$ is the resulting strain in the transverse direction. The value of $\varepsilon_{\perp}$ was calculated for each independent simulation run upon equilibration and was averaged over all the elements. In Figure $\mathrm{S} 6$, we plot the values of $v$ calculated from the simulations as a function of $T$, along with the numerical calculations ${ }^{2}$ as $v=\frac{(3 K-2 G)}{2(3 K+G)}$, where $K$ and $G$ are bulk and shear moduli ${ }^{2-5}$ (formulas are provided in the Model section). Figure S6 shows a good agreement between the analytical predictions and simulation results. Notably, this functional dependence of Poisson's ratio on temperature for thermoresponsive gels undergoing volume phase transition was previously reported in experiments ${ }^{4,6,7}$ and analytical calculations ${ }^{4}$.

C. Linear stability analysis. At small deformations, the linear elasticity can be employed, with the inplane components of the stress tensor $\sigma_{i j}(i=x, y)$ expressed via the components of the strain tensor $\varepsilon_{i j}$ as $^{8}$

$$
\begin{gathered}
\varepsilon_{i j}=\frac{1}{2}\left(\frac{\partial u_{i}}{\partial x_{j}}+\frac{\partial u_{j}}{\partial x_{i}}+\frac{\partial \zeta}{\partial x_{i}} \frac{\partial \zeta}{\partial x_{j}}\right), \\
\sigma_{x x}=\frac{E}{1-v^{2}}\left(\varepsilon_{x x}+v \varepsilon_{y y}\right), \\
\sigma_{y y}=\frac{E}{1-v^{2}}\left(\varepsilon_{y y}+v \varepsilon_{x x}\right), \\
\sigma_{x y}=\frac{E}{1+v} \varepsilon_{x y},
\end{gathered}
$$


where $u_{i}(i=x, y)$ and $\zeta$ describe the in-plane and the out-of-plane displacements, respectively, $v$ is the Poisson's ratio, and $E$ is the Young's modulus. Due to the sample's confinement along the long edges, the sample motion is restricted both along the length and along the width of the sample (with the exception of the swelling along the unrestricted short ends; however, these end effects are not considered in the linear stability analysis). An effect of this confinement can be approximated as an application of an equi-biaxial compressive strain $\varepsilon$ on the free gel at the lower temperature, $T_{f}$, so that the sample reaches the dimensions corresponding to that at the initial higher temperature, $T_{i}$, (Figure S7a). We postulate that a constrained swelling of the gel can be approximated as a free swelling due to the temperature quench from $T_{i}$ to $T_{f}$ followed by the equi-biaxial compressive strain $\varepsilon=\varepsilon_{x x}=\varepsilon_{y y}$. Thereby the compressive strain $\varepsilon$ can be correlated with the characteristic swelling ratio $\alpha$ as $\varepsilon=\alpha^{-1}-1$ (Figure S7a). Hence, the effective compressive stress exerted on the sample can be expressed as

$$
\sigma(\alpha)=\frac{E}{1-v}\left(\frac{1}{\alpha}-1\right)
$$

where $\alpha$ is defined by the depth of the temperature quench (Figure S4).

The out-of-plane displacement $\zeta(x, y)$ describing the topography of an elastic plate with an initial width $w_{0}$ and a constant thickness $h_{0}$ can be found by solving Foppl-von Karman (FvK) equations ${ }^{8,9}$ :

$$
\begin{gathered}
D_{E} \Delta^{2} \zeta-h \sigma\left(\frac{\partial^{2} \zeta}{\partial x^{2}}+\frac{\partial^{2} \zeta}{\partial y^{2}}\right)=0, \\
\frac{\partial \sigma_{i j}}{\partial x_{j}}=0,
\end{gathered}
$$

where $\Delta^{2}=\frac{\partial^{2}}{\partial x^{2}}+2 \frac{\partial^{2}}{\partial x \partial y}+\frac{\partial^{2}}{\partial y^{2}}$ and $D_{E}=\frac{E h_{0}{ }^{3}}{12\left(1-v^{2}\right)}$ is the bending stiffness. The Young's modulus and the Poisson's ratio depend on the sample temperature (see Section $\mathrm{S} 1$ : B). The solution of $\zeta(x, y)$ has a general form of 9,10

$$
\zeta(x, y)=\left(C_{1} e^{-\eta y}+C_{2} e^{\eta y}+C_{3} \cos (\beta y)+C_{4} \sin (\beta y)\right) \sin (k x),
$$

where $k$ is the wave vector and $C_{i}(i=1,2,3,4)$ are four constants. Substituting eq. s8 into eq. s6 gives $\eta=k$ and $\beta=\sqrt{\sigma / D_{E}-k^{2}}$. The boundary conditions corresponding to the two clamped edges along the $\mathrm{y}-$ direction are $\zeta_{y=0, y=w_{0}}=0$ and $(\partial \zeta / \partial y)_{y=0, y=w_{0}}=0$, respectively. The non-zero solution of four linear equations for $C_{i}(i=1,2,3,4)$ constants exist if the determinant $g(k, \sigma)=$ $\left[\begin{array}{cccc}1 & 1 & 1 & 0 \\ -\eta & \eta & 0 & \beta \\ e^{-\eta w_{0}} & e^{\eta w_{0}} & \cos \left(\beta w_{0}\right) & \sin \left(\beta w_{0}\right) \\ -\eta e^{-\eta w_{0}} & \eta e^{\eta w_{0}} & -\beta \sin \left(\beta w_{0}\right) & \beta \cos \left(\beta w_{0}\right)\end{array}\right]$ is equal to zero. Taking $w_{0}$ and $D_{E} /\left(h_{0} w_{0}{ }^{2}\right)$ as respective units of length and stress ${ }^{9,10}$, we rewrite the determinant as $\bar{g}(\bar{k}, \bar{\sigma})$ and plot the contour of $\bar{g}(\bar{k}, \bar{\sigma})=0$ (Figure S7b). As can be seen from this plot, a dimensionless magnitude of the compressive stress above which the uniform flat state loses its stability is $\bar{\sigma}_{c}=37.08$. The dimensionless wavevector at the onset of the instability is $\bar{k}_{c} \approx 2.4$ and the corresponding wavelength $\lambda_{W}^{*}=\frac{2 \pi}{\bar{k}} w_{0} \approx 2.62 w_{0}$ (plotted as the dashed line in Figure $2 a)$. We note that while a similar analysis was originally performed ${ }^{9}$ and then later utilized ${ }^{10}$ for the hydrogels with one clamped edge undergoing swelling, this is the first time this approach is adapted to the samples with both edges clamped. Due to the different boundary conditions, an analytical estimate above gives a lower value of the pattern wavelength than that estimated analytically and observed for the patterns with one clamped edge ${ }^{9}, \lambda_{W}^{*} \approx 3.26 w_{0}$. Note that the pattern wavelength is defined solely by the sample geometry, while the elastic properties of the sample (the Young's modulus and the Poisson's ratio) need to be taken into account along with sample's dimensions to estimate a critical stress based on the above critical value as $\sigma_{c}=\frac{37.08 D_{E}}{h_{0} w_{0}{ }^{2}}$. 
Finally, the above solution allows us to identify a critical characteristic swelling ratio $\alpha_{c}$ (which in turn is defined by the depth of the temperature quench), defining an onset of instability. Equating $\sigma_{c}$ to the magnitude of the compressive stress, $\sigma(\alpha)$ (eq. s5), gives an analytical estimate of the critical value of $\alpha_{c}$ corresponding to the onset of instability as:

$$
\alpha_{c}^{*}=\left[1-\frac{3.09}{1+v}\left(\frac{h_{0}}{w_{0}}\right)^{2}\right]^{-1}
$$

This value is plotted in Figure $2 \mathrm{~b}$ for the Poisson's ratio $v=0.14$. The low values of the Poisson's ratio for swollen gels close to the volume phase transition temperatures were reported in previous experiments, 6,7 . While for simplicity we fix $v$ in Figure $2 \mathrm{~b}$, varying this value within the range reported in Figure $6 \mathrm{~b}$ insignificantly affects the curve in Figure $2 \mathrm{~b}$. Comparison of eq. $\mathrm{s} 9$ with the similar expression derived for the samples with the single edge clamped ${ }^{9,11}, \alpha_{c}^{*} \approx 1+0.87\left(\frac{h_{0}}{w_{0}}\right)^{2}$ indicates that significantly higher critical swelling ratio $\alpha_{c}^{*}$ is required to observe the onset of buckling for the samples of the same dimensions but with both long edges clamped.

\section{Section S2: Supplementary figures}

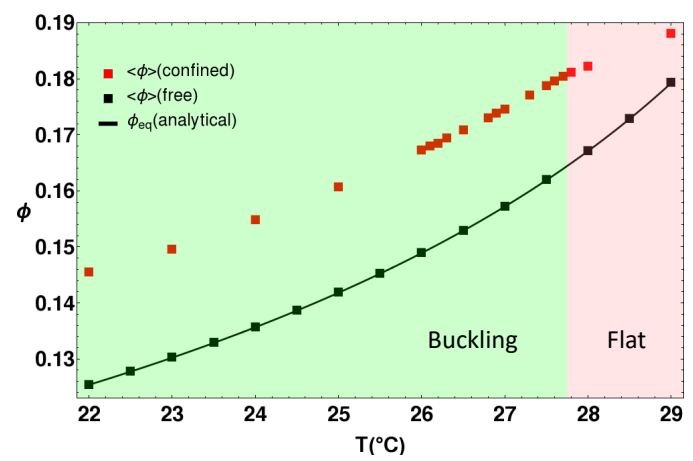

Figure S1. Equilibrium volume fraction of polymer, $\phi$, as a function of temperature, $T$. The samples which are either freely swollen in solvent (black symbols) or constrained along the long edges (red symbols) undergo instantaneous temperature quench from $30^{\circ} \mathrm{C}$ to the temperatures specified in the $x$-axis. The $\langle\phi\rangle$ is the volume fraction of the polymer averaged over all the elements within the sample upon reaching an equilibrium. Red symbols within the green region represent buckled constrained samples. All free samples remain flat in equilibrium. System parameters correspond to a reference case as listed in the Model section (dimensionless sample size $87.0 \times 11.7 \times 1.7$ and $c_{0}=4 \times 10^{-3}$ ). 

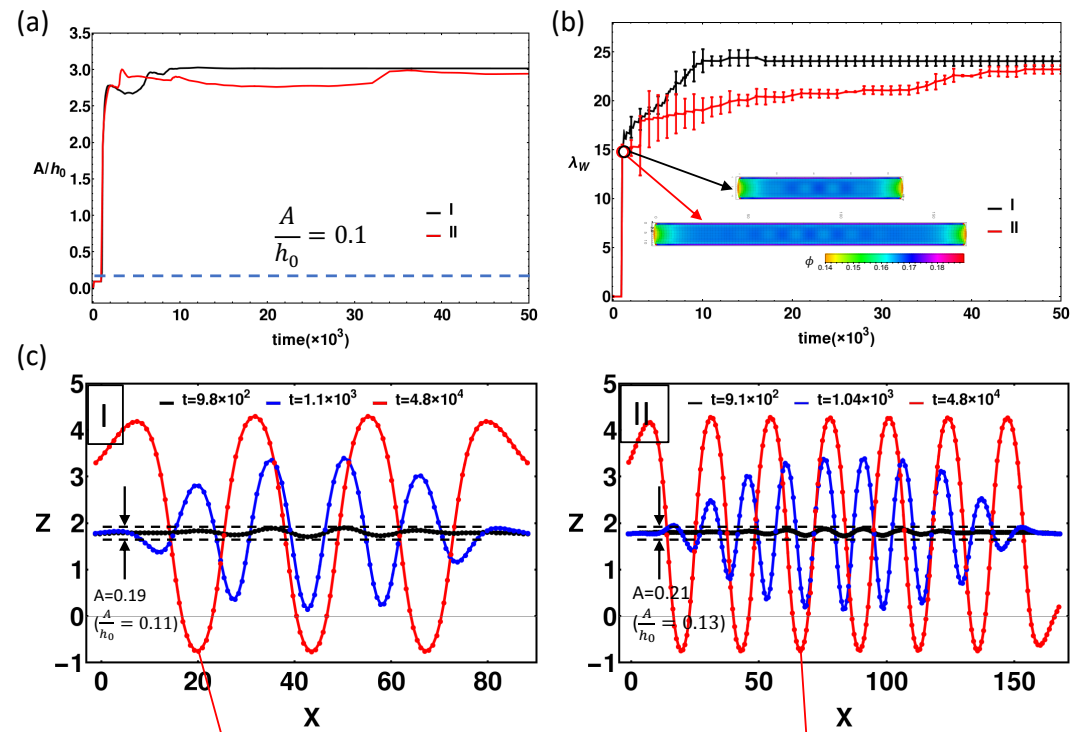

(d)
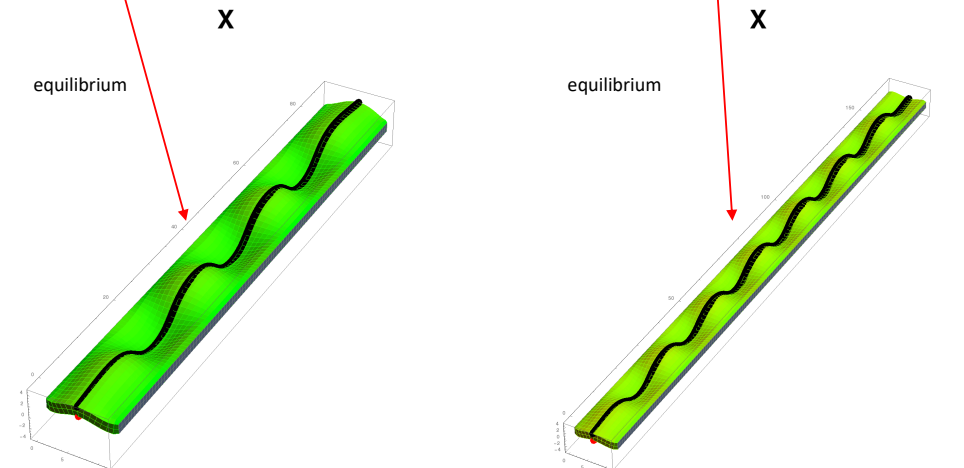

Figure S2. Time evolution of patterns upon the temperature quench. The sample I is the reference case (the same simulation as in Figure 1a-c); at $t=0$ the sample undergoes a temperature quench from $30^{\circ} \mathrm{C}$ to $24^{\circ} \mathrm{C}$. All the parameters in the sample II are identical to those in the reference case, except the sample length is extended (dimensionless size is $166.5 \times 11.7 \times 1.7$ ). (a) The undulations of the top surface, $A$, normalized on the initial thickness, $h_{0}$. An undulation $A$ is defined as the distance between the highest and the lowest $z$-coordinates of the upper face taken at the middle node along the sample width (marked by blue curve in (d)). (b) Time evolution of the wavelength, $\lambda_{W}$ (note that $\lambda_{W}$ is calculated if $A / h_{0} \geq 0.1$ ). (c) Vertical coordinates of the middle of the top face in cases I and II as patterns develop with time (dimensionless simulation times are provided in legends). (d) The morphologies of samples at equilibrium. 

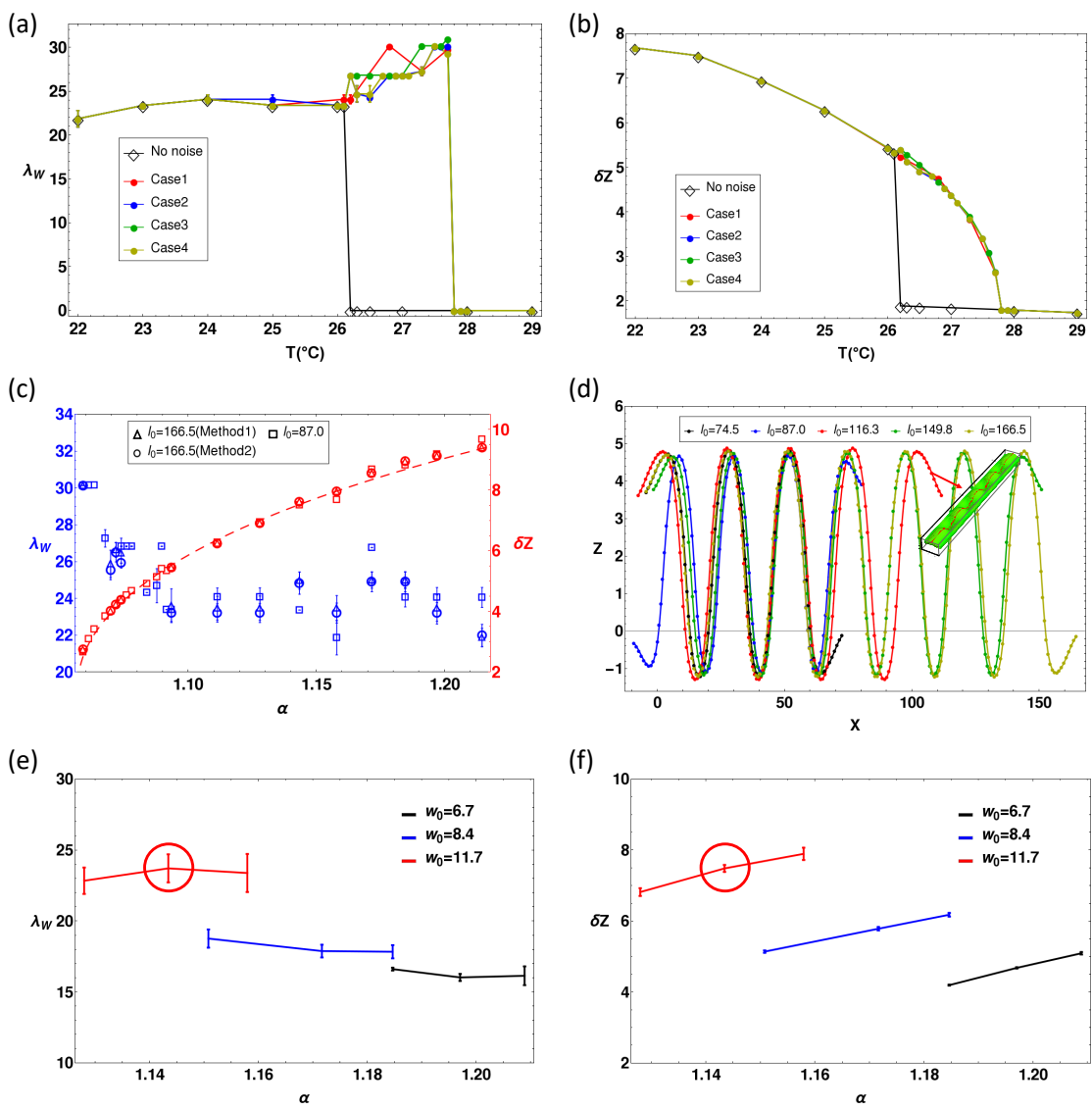

Figure S3. Effects of random perturbations and sample length. (a) The wavelength $\lambda_{W}$ and (b) the amplitude $\delta Z$ of the fully developed patterns in the constrained gel membranes under various random perturbations (Cases 1-4 are detailed in Section S1: A). The reference parameters are chosen, including the dimensionless initial size $87.0 \times 11.7 \times 1.7$, the crosslink density $c_{0}=4.0 \times 10^{-3}$, the volume fraction of polymer at preparation $\phi_{0}=0.114$, and the initial temperature $T_{i}=30^{\circ} \mathrm{C}$. (c) The equilibrium wavelength $\lambda_{W}$ and the amplitude $\delta Z$ of patterns for the samples with two different lengths listed in the legend as a function of the swelling ratio $\alpha$. All other parameters are the same as in (a). The red curve is the best fit using eq. 4 with $p_{1}=22.46$ and $p_{2}=1.57$; herein, $\alpha_{c}=1.057$. We characterize patterns in the longest samples $\left(l_{0}=166.5\right)$ using all the peaks on the top surface $(\Delta)$ and excluding the peaks nearest to both ends $(\square)$, the second method is used in calculations of the wavelength in all the cases of the longest sample bellow. For the shorter samples, all the peaks are used while calculating the wavelength. (d) Vertical coordinates of the middle of the top face of the samples at equilibrium (along the red line in the inset), samples' lengths are listed in the legend, remaining parameters are: $w_{0}=11.7, c_{0}=4 \times 10^{-3}, T_{i}=30^{\circ} \mathrm{C}$, and $T_{f}=23^{\circ} \mathrm{C}$. (e) The wavelength $\lambda_{W}$ and (f) the amplitude $\delta Z$ of buckled samples equilibrated at the temperatures corresponding to respective values of $\alpha$; each point represents the average value calculated at the same conditions for four lengths, including $l_{0}=74.5,87.0,116.3$, and 149.8. The data points that correspond to the samples in (d) are marked by the red circles in (e) and (f). 


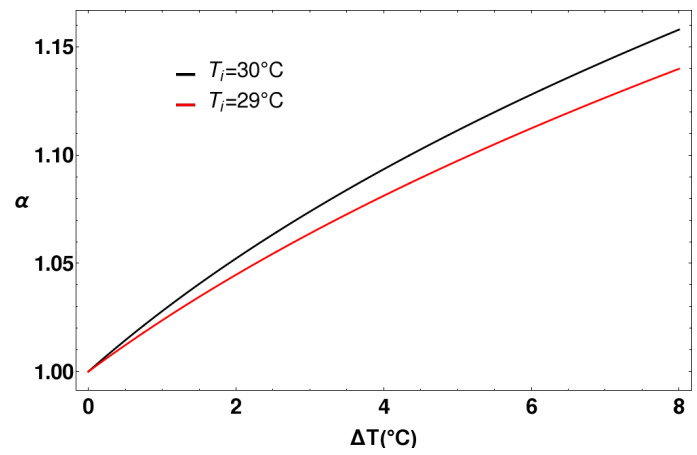

Figure S4. Characteristic ratio $\alpha$ as a function of the depth of the temperature quench, $\Delta T=\left|T_{f}-T_{i}\right| . \alpha$ is defined as the ratio of the degrees of swelling of the free sample at the final temperature $T_{f}$ to that at the initial temperature $T_{i}$ as $\alpha=\lambda_{e q}\left(T_{f}\right) / \lambda_{e q}\left(T_{i}\right)$.
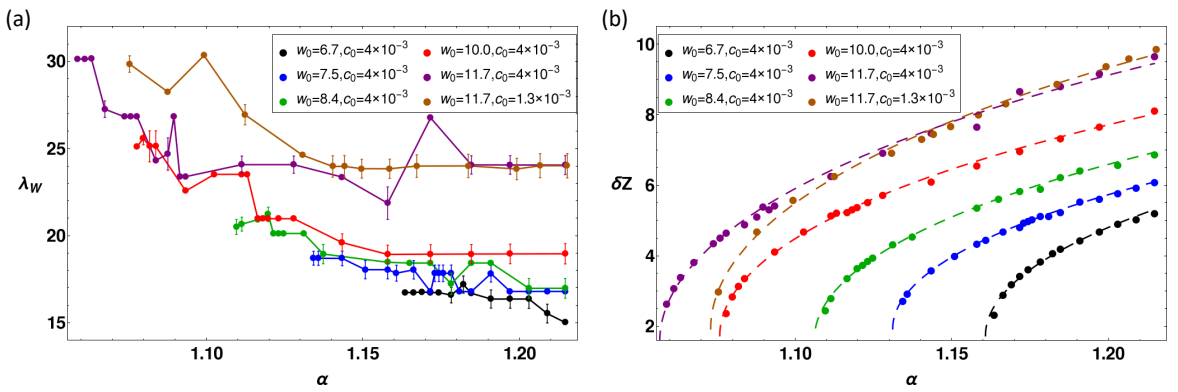

Figure S5. Effects of the sample width and crosslink density on the wavelength and the amplitude. Dependence of (a) the wavelength $\lambda_{W}$ and (b) the amplitude $\delta Z$ of the confined gels with various widths $w_{0}$ and crosslink densities $c_{0}$ as shown in the legend on the ratio $\alpha$. The initial temperature is $T_{i}=30^{\circ} \mathrm{C}$. The initial length and thickness of the gels with $c_{0}=4.0 \times 10^{-3}$ are $l_{0}=87.0$ and $h_{0}=1.7$, respectively. The initial size of the samples with $c_{0}=1.3 \times 10^{-3}$ is $86.9 \times 11.7 \times 2.0$. The dashed lines in (b) represent the fitting (eq. 4), with $\alpha_{c}$ and the fitting parameters $p_{1}$ and $p_{2}$ listed in Table S1.
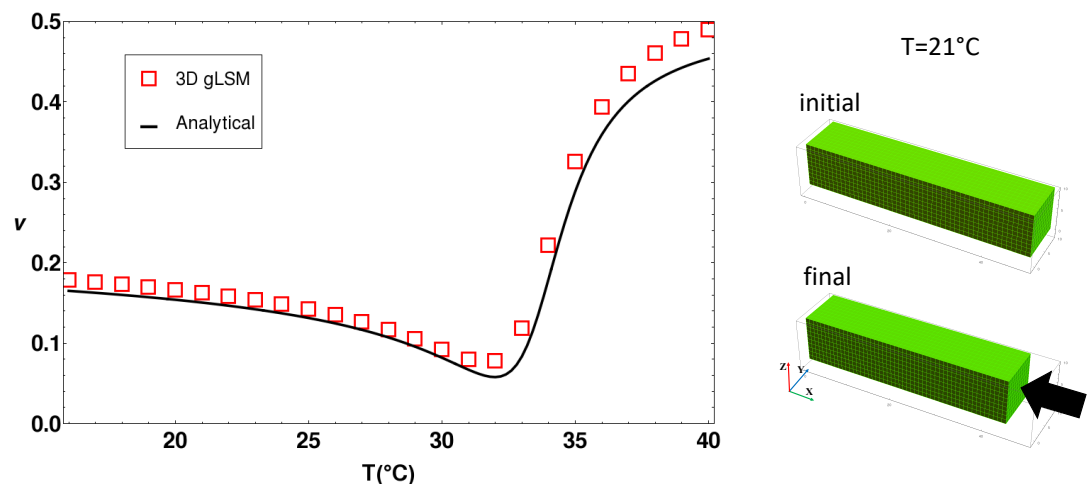

Figure S6. Poisson's ratio of PNIPAAm gels. Dependence of the Poisson's ratio on temperature. Red open squares $(\square)$ correspond to the independent simulation runs at each temperature under the uniaxial compression (as marked by the arrow in the image on the right corresponding to a chosen temperature $T=$ $21^{\circ} \mathrm{C}$ ) with a compressive strain $\varepsilon_{\|}=0.1$. Black curve corresponds to the analytical calculation of the Poisson's ratio (Section S1: B). Parameters of the gel are set in both simulations and analytical calculations 
as $\phi_{0}=0.114$ and $c_{0}=4 \times 10^{-3}$, the sample size is at $51 \times 11 \times 11$ nodes, remaining details are given in Section S1: B.

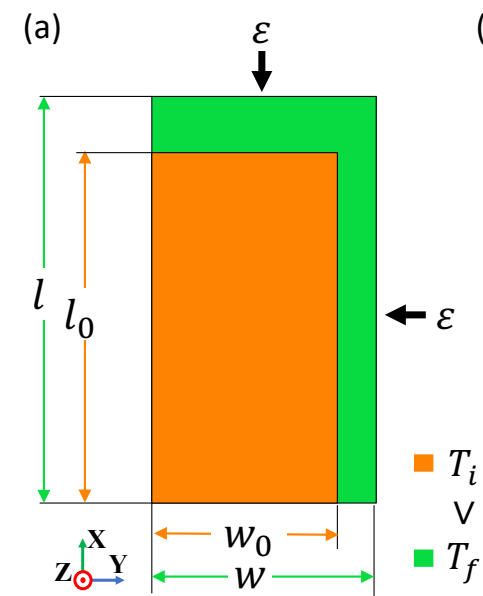

(b)

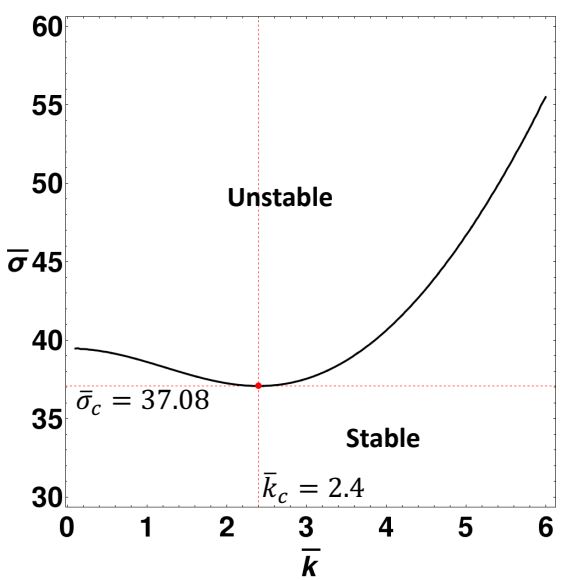

Figure S7. Linear stability analysis of the flat confined elastic plate. (a) Schematics of the thin unconfined elastic plate at initial high temperature (orange) and at a lower temperature (green). The dimensions of the free gel at the higher temperature $T_{i}$ and at the lower temperature $T_{f}$ are $l_{0} \times w_{0}$ and $l \times w$, respectively. An application of an equi-biaxial compressive strain $\varepsilon$ on the free gel at the lower temperature $\left(T_{f}\right)$ effectively results in the change of the dimensions from $l \times w$ to $l_{0} \times w_{0}$. Recall that $\alpha$ is defined as a ratio of the degrees of swelling of a free sample at respective temperatures, $\alpha \equiv w / w_{0} \equiv l / l_{0}$; hence effective compressive strain can be defined as $\varepsilon=\alpha^{-1}-1$. (b) The $\bar{\sigma}, \bar{k}$ phase space for the stability of equi-biaxially compressed plate. The red $\operatorname{dot}\left(\bar{k}_{c}=2.4, \bar{\sigma}_{c}=37.08\right)$ represents the critical point; above $\bar{\sigma}_{c}$ the flat state loses its stability. Details are provided in Section S1: C.

(a)

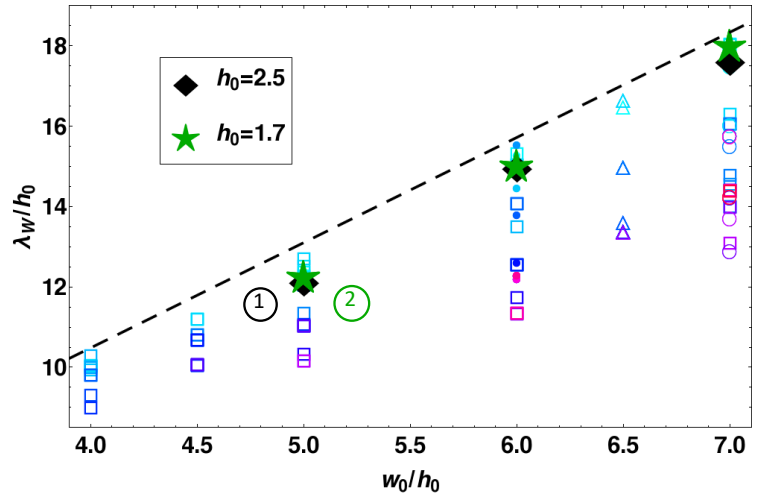

(b)

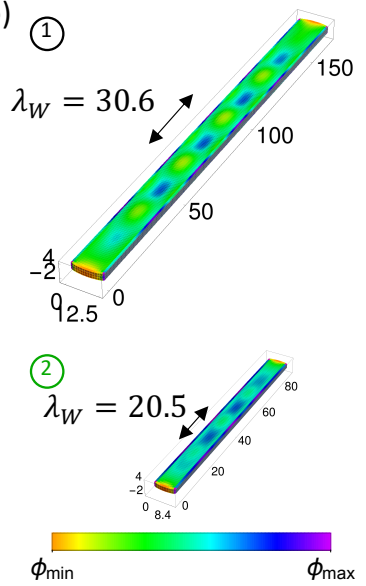

Figure S8. Effects of the sample thickness. (a) The wavelength $\lambda_{W}$ at equilibrium as a function of the initial width $w_{0}$. Both $\lambda_{W}$ and $w_{0}$ are normalized by the initial thickness of the sample at $T_{i}, h_{0}$. The background image is taken from Figure $2 \mathrm{a}$, the data points for the thicker sample $(\diamond)$ are added to this image. The thicker samples $(\diamond)$ are initially equilibrated at $T_{i}=30^{\circ} \mathrm{C}$ with initial sizes of $166.5 \times 12.5 \times 2.5\left(w_{0} / h_{0}=5\right), 166.5 \times 15.1 \times 2.5\left(w_{0} / h_{0}=6\right)$, and $166.5 \times 12.5 \times 2.5\left(w_{0} / h_{0}=\right.$ $7)$, respectively. The depth of the temperature quench is chosen to correspond to that in the cases with reference sample thickness $(\star)$ in this image. The corresponding final temperatures, $T_{f}$, are 25.1, 26.8, 
and $27.7^{\circ} \mathrm{C}$, respectively, for the three chosen ratios of $w_{0} / h_{0}$. (b) The morphologies of two samples of different thickness as marked in (a). The color represents the local values of polymer volume fraction according to the color bar with $\phi_{\min }=0.167$ and $\phi_{\max }=0.194$ (top image in b), and $\phi_{\min }=0.147$ and $\phi_{\max }=0.185$ (bottom image in b).

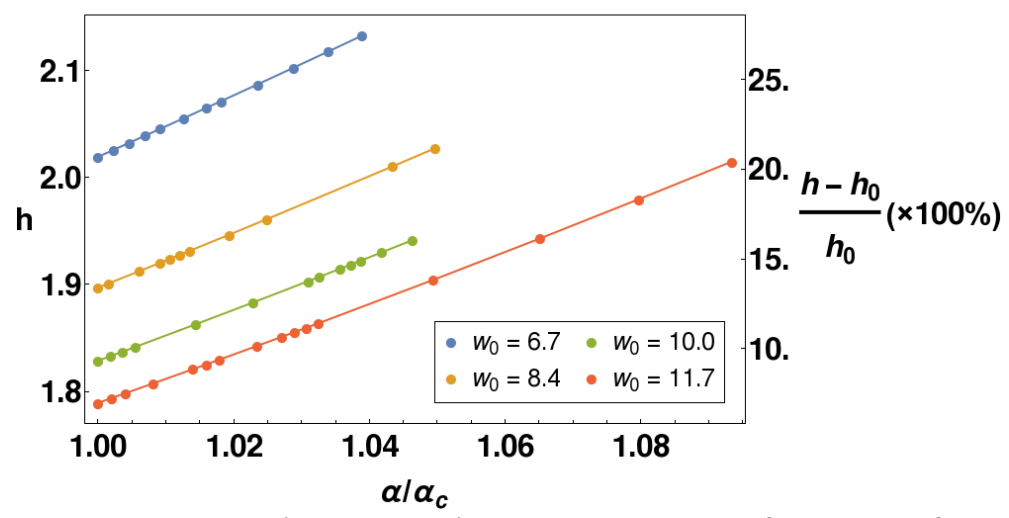

Figure S9. The average sample thickness prior to the onset of pattern formation. The samples (crosslink density $c_{0}=4 \times 10^{-3}$, initial length $l_{0}=87.0$, and initial thickness $h_{0}=1.7$ ) with various widths as listed in the legend are subjected to the temperature quench of various depth (same data as in Figure 2, initial temperature $T_{i}=30^{\circ} \mathrm{C}$ ). The sample thickness $h$ averaged over the entire sample is calculated prior to buckling. The right axis shows the corresponding relative variation in the sample thickness (in \%) due to the sample swelling.

(a)

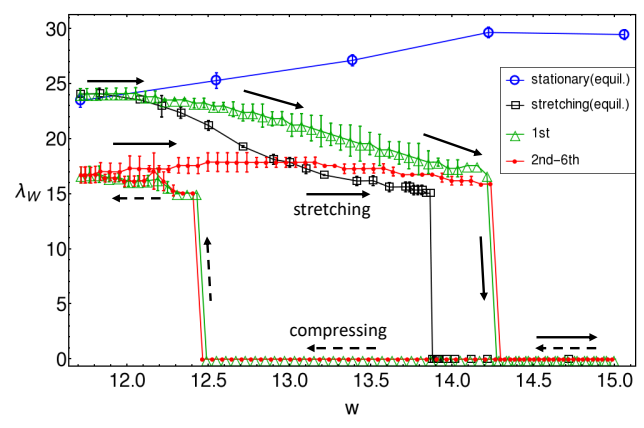

(b)

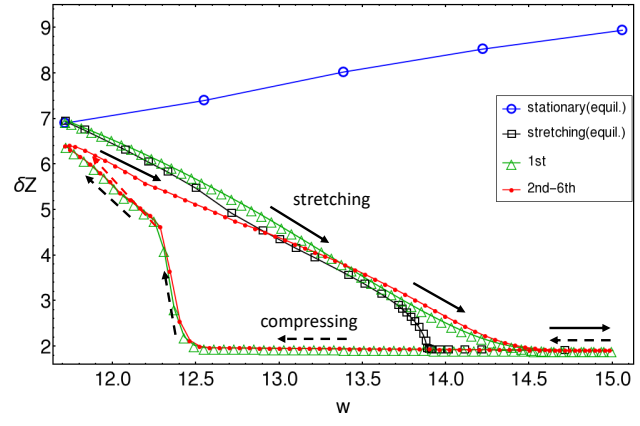

(c)

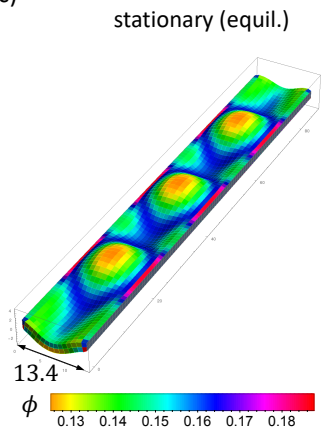

stretching (1st)

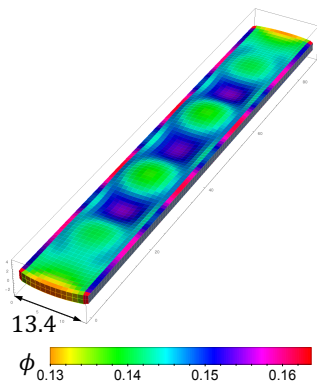

stretching (equil.)

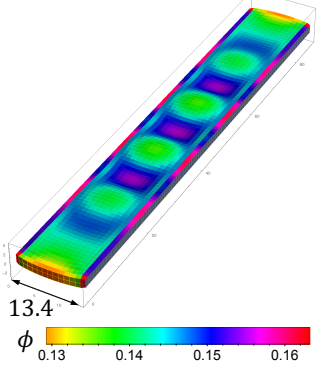

stretching (2nd)

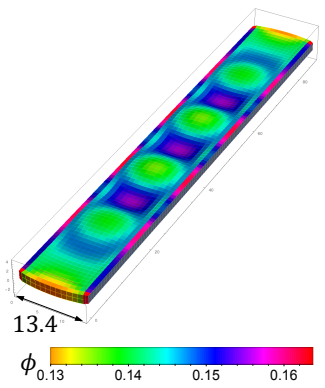

Figure S10. Patterns characteristics under stretching and compression cycles. (a) The wavelength and (b) the amplitude as functions of the sample's width during different stretching and compressing cycles as given in the legend. Six stretching and compression cycles are used, both characteristics during the cycles 2-6 are identical (within the error bars) in (a) and (b). The wavelength and the amplitude of the patterns in samples with fixed boundaries and matched widths are also characterized (blue open symbols in (a) and (b) 
marked by "stationary(equil.)"). The wavelength and the amplitude of the patterns from the additional series of simulations, where the samples with initial width $w_{0}=11.7$ are stretched to various widths in independent runs and attain an equilibrium after an initial temperature quench from $30^{\circ} \mathrm{C}$ to $24^{\circ} \mathrm{C}$, are shown by the black squares and denoted as "stretching (equil.)" (c) The morphologies of the samples at different conditions as listed. The color represents the local volume fraction of polymer $\phi$. The reference parameters are used (same as in Figure 3, including the crosslink density $c_{0}=4 \times 10^{-3}$, the initial temperature $T_{i}=30^{\circ} \mathrm{C}$, and the initial size of sample under stretching and compression cycles $87.0 \times 11.7 \times 1.7)$.

(a)

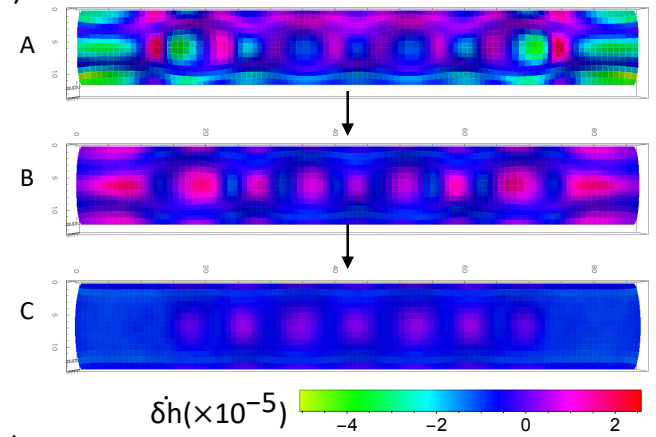

(c)

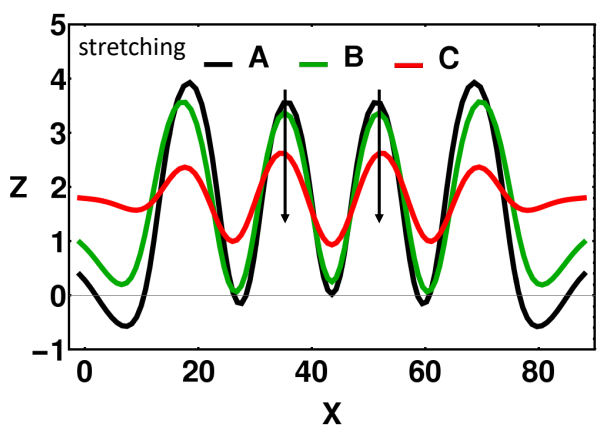

(b)

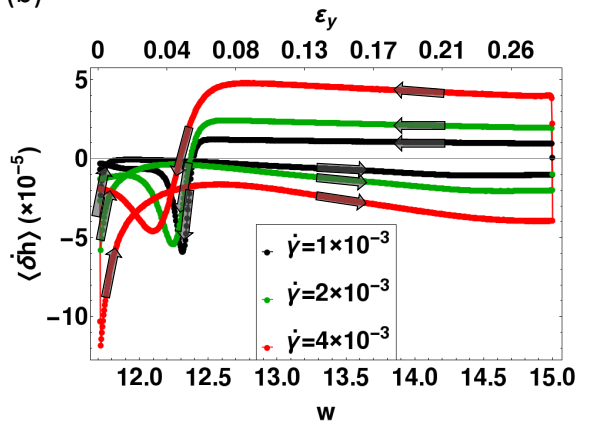

(d)

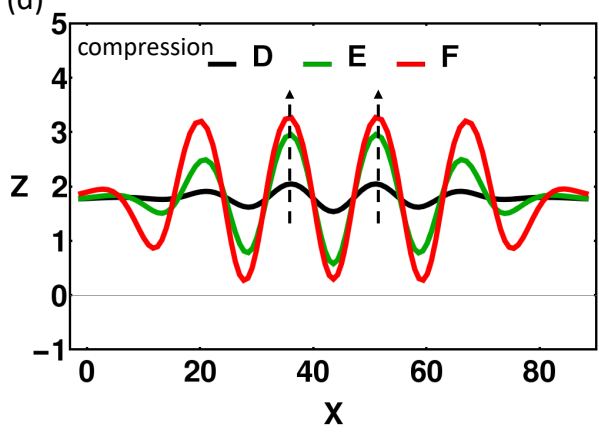

Figure S11. Dynamics of patterns during an equilibrated cycle. An additional analysis of a stretching and compression cycle depicted in Figure $4\left(\dot{\gamma}=1 \times 10^{-3}\right)$, the A-F states correspond to the time instances marked in Figure 3a. (a) The morphologies of the sample during stretching (A-C states as marked in (a), top view), with color representing the local value of $\dot{\delta} h$. (b) The rate of change of the effective average thickness $\langle\dot{\delta h}\rangle$ during the stretching and compression cycle at three rates as listed in the legend. The reference parameters are used (same as in Figure 3, including the crosslink density $c_{0}=4 \times 10^{-3}$, the initial temperature $T_{i}=30^{\circ} \mathrm{C}$, and the initial size of the sample $\left.87.0 \times 11.7 \times 1.7\right)$. (c-d) The vertical coordinates of the middle (y-direction) of the top face of the sample during stretching (in (c)) and compression (in (d)). The arrows point out the direction of motion of the peaks. 
(a)

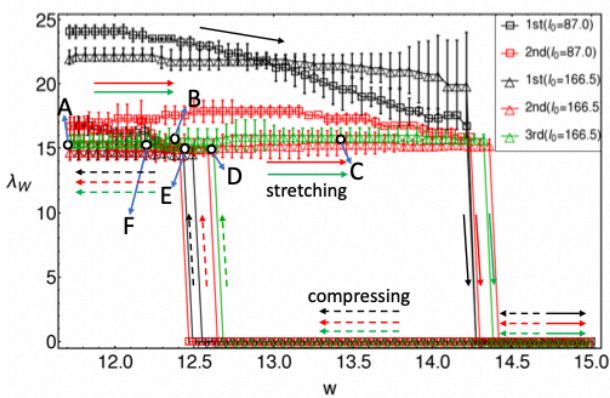

(c)

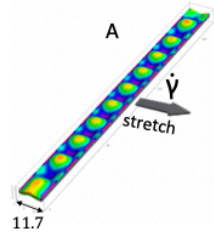

(d)
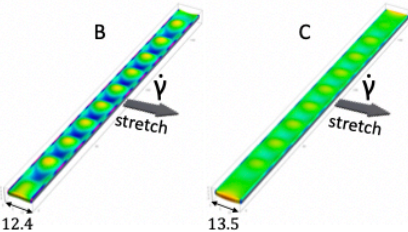

(e)

(b)
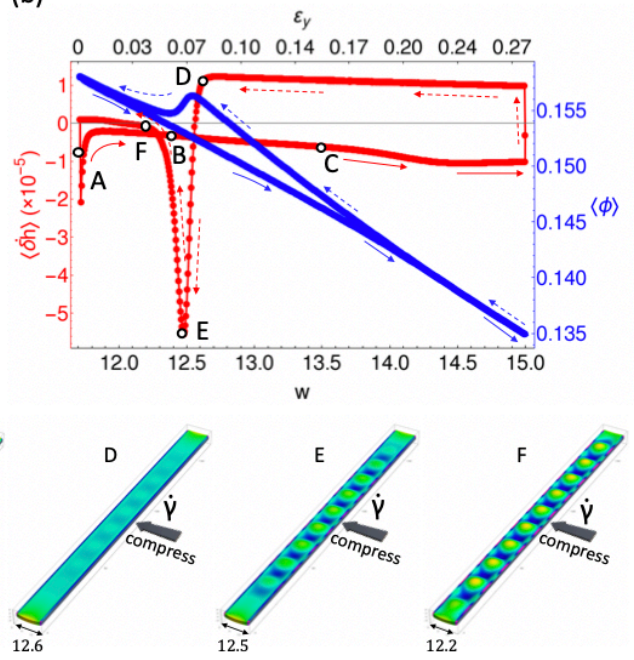

$\phi_{\min }^{0.13} 0.140 .150 .160 .170 .18 \phi_{\max }^{0.19}$

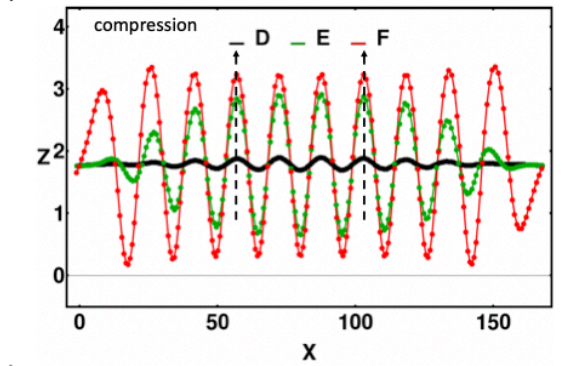

(g)
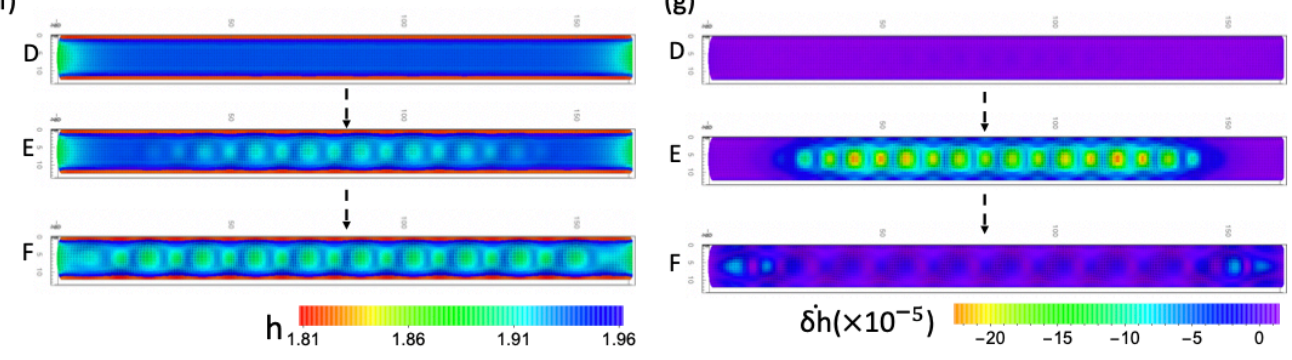

Figure S12. Effects of the sample length. The same parameters $\left(c_{0}=4 \times 10^{-3}\right.$, the initial temperature $T_{i}=30^{\circ} \mathrm{C}$, the initial width and thickness $11.7 \times 1.7$, and the strain rate $\dot{\gamma}=1 \times 10^{-3}$ ) are chosen as in the example in Figures 3, but the length of sample is extended to $l_{0}=166.5$. (a) The wavelength as a function of width, $w$, during $1^{\text {st }}, 2^{\text {nd }}$, and $3^{\text {rd }}$ stretching and compression cycles. The $3^{\text {rd }}-6^{\text {th }}$ cycles are identical, while there are small differences between the $2^{\text {nd }}$ and $3^{\text {rd }}$ cycles unlike in the case of the shorter sample. We exclude the peaks near the ends to characterize the patterns in the longer samples. (b) The rate of change in the effective average thickness $\langle\dot{\delta h}\rangle$ (red, left axis) and the average volume fraction of polymer $\langle\phi\rangle$ (blue, right axis) during an equilibrated stretching and compression cycle. The A-F states correspond to the same states marked in (a). The directions of stretching and compression are indicated by the solid and dashed arrows, respectively. (c) Sample morphology within a single $\left(5^{\text {th }}\right)$ cycle (A-C states as marked in (a) and (b)), with color representing the local value of $\langle\phi\rangle$. (d-e) The vertical coordinates of the middle node (y-direction) of the top face of the sample during stretching (A-C states, in (d)) and compression (DF states, in (e)). The arrows point out the direction of motion of peaks. (f-g) Sample morphology during the compression (D-F states as marked in (a), top view), with color representing the local thickness, $h$, in (f), and the local value of $\dot{\delta} h$ in $(\mathrm{g})$. 

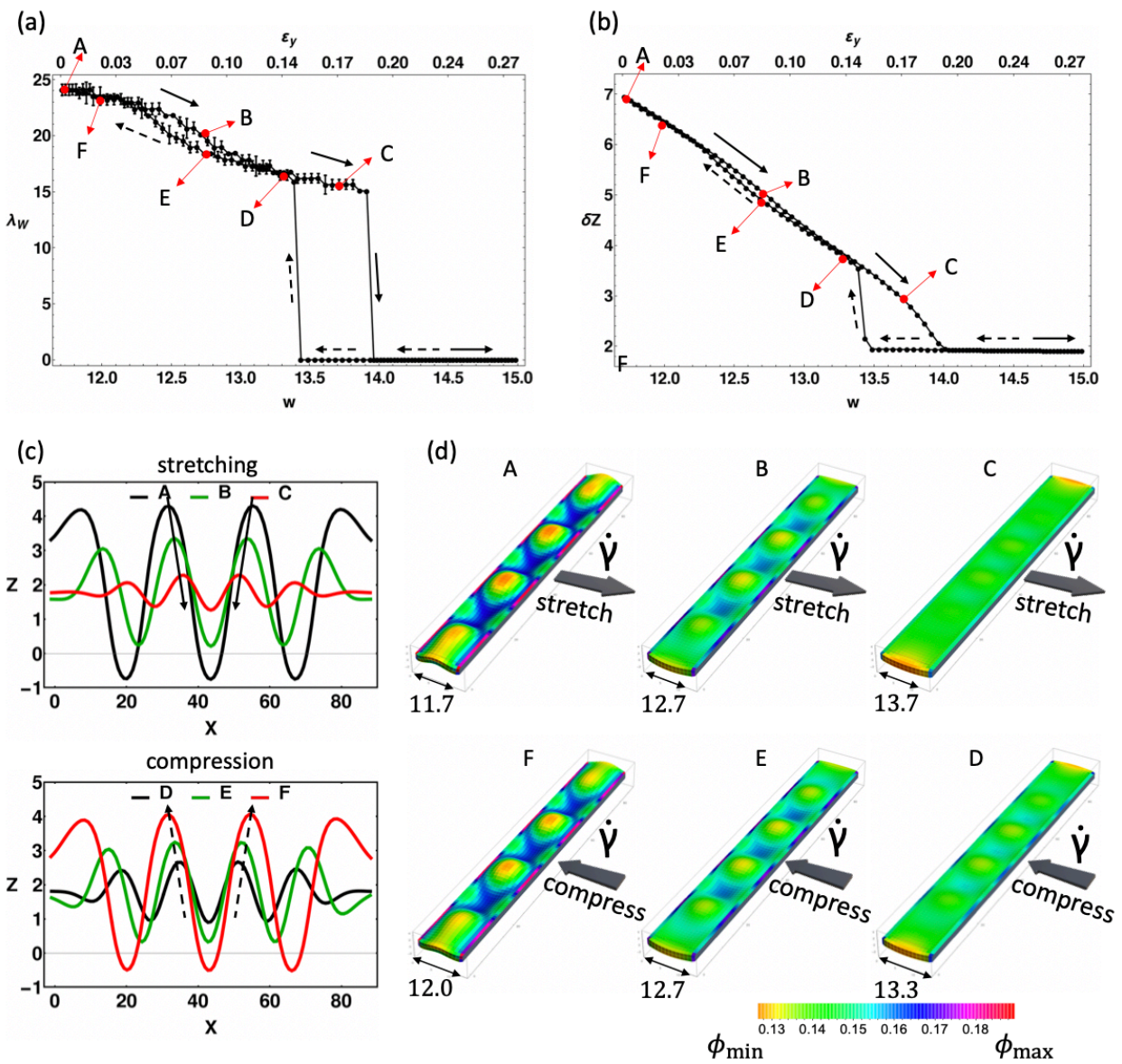

Figure S13. Dynamics during slow stretching and compression $\left(\dot{\gamma}=5 \times 10^{-5}\right)$. (a) The wavelength and (b) the amplitude as functions of width, the directions of stretching and compression are indicated by the solid and dashed arrows, respectively. The dynamics is identical within all the cycles $\left(1^{\text {st }}-6^{\text {th }}\right)$ (c) The vertical coordinates of the middle of the top face of the sample during the stretching (top) and compression (bottom). The arrows indicate the direction of motion of the peaks. The A-F states are marked in (a) and (b). (d) The morphologies of the sample at A-F states, blue arrows show the direction of the motion of the boundary and color represents the local polymer volume fraction according to the color bar. The reference parameters are used (the crosslink density $c_{0}=4 \times 10^{-3}$, the initial temperature $T_{i}=30^{\circ} \mathrm{C}$, and the initial size $87.0 \times 11.7 \times 1.7)$.

\section{Section S3: Supplementary Tables}

Table S1. Values of $\alpha_{c}, p_{1}$, and $p_{2}$ for dashed fitting curves in Figure S5b.

\begin{tabular}{ccccc}
$\boldsymbol{w}_{\mathbf{0}}$ & $\boldsymbol{c}_{\mathbf{0}}$ & $\boldsymbol{\alpha}_{\boldsymbol{c}}$ & $\boldsymbol{p}_{\mathbf{1}}$ & $\boldsymbol{p}_{\mathbf{2}}$ \\
\hline 6.7 & $4.0 \times 10^{-3}$ & 1.161 & 18.28 & 1.74 \\
\hline 7.5 & $4.0 \times 10^{-3}$ & 1.131 & 16.88 & 1.93 \\
8.4 & $4.0 \times 10^{-3}$ & 1.106 & 18.60 & 1.65 \\
10.0 & $4.0 \times 10^{-3}$ & 1.076 & 19.29 & 1.74 \\
11.7 & $4.0 \times 10^{-3}$ & 1.057 & 22.46 & 1.57 \\
11.7 & $1.3 \times 10^{-3}$ & 1.073 & 23.58 & 1.93
\end{tabular}




\section{References}

1. Kuksenok, O.; Yashin, V. V.; Balazs, A. C., Global signaling of localized impact in chemoresponsive gels. Soft Matter 2009, 5 (9), 1835-1839.

2. $\quad$ Doi, M., Soft Matter Physics. Oxford University Press: 2013; p 272.

3. Rubinstein, M.; Colby, R. H., POLYMER PHIYSICS. Oxford University Press: 2003; p 454.

4. Hirotsu, S., Softening of Bulk Modulus and Negative Poisson Ratio near the Volume PhaseTransition of Polymer Gels. J Chem Phys 1991, 94 (5), 3949-3957.

5. $\quad$ Treloar, L. R. G., The Physics of Rubber Elasticity. 3 edition ed.; Oxford University Press: 2005; p 310 .

6. Greaves, G. N.; Greer, A. L.; Lakes, R. S.; Rouxel, T., Poisson's ratio and modern materials. Nat Mater 2011, 10 (11), 823-837.

7. Voudouris, P.; Florea, D.; van der Schoot, P.; Wyss, H. M., Micromechanics of temperature sensitive microgels: dip in the Poisson ratio near the LCST. Soft Matter 2013, 9 (29), 7158-7166.

8. $\quad$ Landau, L. D.; Lifshitz, E. M., Theory of Elasticity. 3 ed.; Butterworth-Heinemann: 1986; p 196. 9. $\quad$ Mora, T.; Boudaoud, A., Buckling of swelling gels. Eur Phys J E 2006, 20 (2), 119-124.

10. Jiang, R. Q.; Xiao, J. L.; Song, J. Z., Buckling of thin gel strip under swelling. Theor Appl Mech Lett 2017, 7 (3), 134-137.

11. DuPont, S. J.; Cates, R. S.; Stroot, P. G.; Toomey, R., Swelling-induced instabilities in microscale, surface-confined poly(N-isopropylacryamide) hydrogels. Soft Matter 2010, 6 (16), 38763882. 